\title{
Mitochondrial hepato-encephalopathy due to deficiency of QIL1/MIC13 (C19orf70), a MICOS complex subunit
}

\author{
Avraham Zeharia ${ }^{1}$, Jonathan R Friedman ${ }^{2}$, Ana Tobar ${ }^{3}$, Ann Saada ${ }^{4}$, Osnat Konen ${ }^{5}$, Yacov Fellig ${ }^{6}$, \\ Avraham Shaag ${ }^{4}$, Jodi Nunnari ${ }^{2}$ and Orly Elpeleg ${ }^{\star, 4}$
}

The mitochondrial inner membrane possesses distinct subdomains including cristae, which are lamellar structures invaginated into the mitochondrial matrix and contain the respiratory complexes. Generation of inner membrane domains requires the complex interplay between the respiratory complexes, mitochondrial lipids and the recently identified mitochondrial contact site and cristae organizing system (MICOS) complex. Proper organization of the mitochondrial inner membrane has recently been shown to be important for respiratory function in yeast. Here we aimed at a molecular diagnosis in a brother and sister from a consanguineous family who presented with a neurodegenerative disorder accompanied by hyperlactatemia, 3-methylglutaconic aciduria, disturbed hepatocellular function with abnormal cristae morphology in liver and cerebellar and vermis atrophy, which suggest mitochondrial dysfunction. Using homozygosity mapping and exome sequencing the patients were found to be homozygous for the p.(Gly15Glufs*75) variant in the QIL1/MIC13 (C19orf70) gene. QIL1/MIC13 is a constituent of MICOS, a six subunit complex that helps to form and/or stabilize cristae junctions and determine the placement, distribution and number of cristae within mitochondria. In patient fibroblasts both MICOS subunits QIL1/MIC13 and MIC10 were absent whereas MIC60 was present in a comparable abundance to that of the control. We conclude that QIL1/MIC13 deficiency in human, is associated with disassembly of the MICOS complex, with the associated aberration of cristae morphology and mitochondrial respiratory dysfunction. 3Methylglutaconic aciduria is associated with variants in genes encoding mitochondrial inner membrane organizing determinants, including TAZ, DNAJC19, SERAC1 and QIL1/MIC13.

European Journal of Human Genetics (2016) 24, 1778-1782; doi:10.1038/ejhg.2016.83; published online 3 August 2016

\section{INTRODUCTION}

Mitochondria are double membrane organelles that perform many essential functions in cells, most notably the production of ATP via oxidative phosphorylation. Mitochondrial function is linked to its form. Although mitochondria are dynamic within cells, they also possess highly organized inner membrane ultrastructure. The mitochondrial inner membrane (IMM) is exceptionally protein dense in nature as a consequence of the abundance of resident respiratory complexes required for oxidative phosphorylation. The IMM possesses distinct morphological and compositionally distinct subdomains, including the inner boundary membrane (IBM) and cristae membranes. The IBM is tightly apposed to the outer mitochondrial membrane and contains the mitochondrial import and assembly machineries. The cristae membranes are lamellar structures that are invaginated into the mitochondrial matrix and contain the respiratory chain complexes. ${ }^{1,2}$ The cristae are separated from the IBM by narrow tubular openings termed cristae junctions (CJs), which are thought to limit the dynamic distribution of the proteins along the IMM and partition the metabolite content of the intracristal and the intermembrane spaces. $^{3}$
The mechanisms underlying the lateral organization of the inner membrane into subdomains are not completely understood. Recently, however, a protein complex highly conserved from yeast to humans named 'mitochondrial contact site and cristae organizing system' (MICOS), was discovered and implicated in inner membrane structure. ${ }^{4-6}$ Evidence suggests that MICOS helps to form and/or stabilize CJs and to determine the placement, distribution and copy number of cristae within mitochondria. The complex is composed of six conserved core subunits, organized into at least two functionally non-redundant subcomplexes. ${ }^{7-9}$ Recently the Human protein QIL1/MIC13 was identified as the structural ortholog of yeast Mic12 and was shown to be required for MICOS complex stability. ${ }^{8} 10$ Specifically, loss of QIL1/MIC13 in cells leads to destabilization of human MIC10, MIC26 and MIC27 and consequently severe IMM morphological defects. ${ }^{8}$ Here we report that variants in QIL1/MIC13 cause a mitochondrial disease in humans, underscoring the conservation of MICOS in mitochondrial ultrastructure and its importance for mitochondrial function in human physiology.

${ }^{1}$ Department of Day Hospitalization, Schneider Children's Medical Center of Israel, Petah Tiqva, and Sackler Faculty of Medicine, Tel-Aviv University, Tel Aviv, Israel; ${ }^{2}$ Department of Molecular and Cellular Biology, University of California, Davis, CA, USA; ${ }^{3}$ Department of Pathology, Rabin Medical Center, Beilinson Campus, Petach Tikva, and Sackler Faculty of Medicine, Tel Aviv University, Tel Aviv, Israel; ${ }^{4}$ Monique and Jacques Roboh Department of Genetic Research, Hadassah, the Hebrew University Medical Center, Jerusalem, Israel; ${ }^{5}$ Imaging Department, Schneider Children's Medical Center of Israel, Petah Tiqva, and Sackler Faculty of Medicine, Tel-Aviv University, Tel Aviv, Israel; ${ }^{6}$ Department of Pathology, Hadassah, the Hebrew University Medical Center, Jerusalem, Israel

*Correspondence: Professor O Elpeleg, Monique and Jacques Roboh Department of Genetic Research, Hadassah, Hebrew University Medical Center, Jerusalem 91120, Israel. Tel: +972 2 6776391; Fax: +972 2 6777499; E-mail: Elpeleg@hadassah.org.il

Received 3 March 2016; revised 18 April 2016; accepted 7 June 2016; published online 3 August 2016 


\section{PATIENTS AND METHODS}

\section{Patients}

Patient II-1 (Figure 1a) was a female, the first child of consanguineous (first cousins once removed) parents of Moslem origin. The patient was born at 42 weeks of gestation by normal vaginal delivery after an uneventful pregnancy. She was small for gestational age $\left(2600 \mathrm{~g}, 5\right.$ th-10th ${ }^{11}$ percentile), with median length and head circumference; Apgar scores were 9 and 10 at 1 and $5 \mathrm{~min}$, respectively. At the age of 3 months she was first evaluated because of failure to thrive. At that age she was alert, responsive, smiled socially and followed moving objects; the neurological examination was reported normal. On followup examination at 10 months she was still failing to thrive $(6400 \mathrm{~g}<2$ nd percentile, $-2.36 \mathrm{SD}$, length $-67 \mathrm{~cm}$, 5th percentile) and had acquired microcephaly (head circumference $41 \mathrm{~cm},<3 \mathrm{rd}$ percentile, $-2.8 \mathrm{SD}$ ). The neurological examination was grossly abnormal with lack of voluntary movements, truncal hypotonia, head lag, limb spasticity, brisk peripheral tendon reflexes and positive Babinski sign. She did not focus her gaze and fundoscopy disclosed bilateral optic atrophy. Hearing could not be tested but brainstem evoked response audiometry was normal. Brain MRI at 1 year of age revealed cerebellar and vermis atrophy (Figures $1 \mathrm{~b}$ and $\mathrm{c}$ ). Other systems were not involved and echocardiography was normal. The patient remained vegetative and was fed through a gastrostomy. At 2.5 years she presented with myoclonic seizures which were refractory to therapy and were the cause of her death at 5 years.

The third child in the family, a male (patient II-3), had an essentially similar course. The pregnancy, birth, Apgar score and growth parameters were normal with median head circumference $(33.8 \mathrm{~cm}, 25$ th percentile) at birth. At 7 months he was referred for evaluation of marked generalized muscle hypotonia. On examination, he was alert and responsive, smiled socially, focused and followed moving objects. Generalized hypotonia and head lag were evident and the tendon reflexes were brisk. The weight was $6700 \mathrm{~g}$ (5th percentile) but head circumference dropped to $<3$ rd percentile, $-3.3 \mathrm{SD}(40 \mathrm{~cm})$. Neurological deterioration was striking at 10 months, when the patient was non-communicative, had no
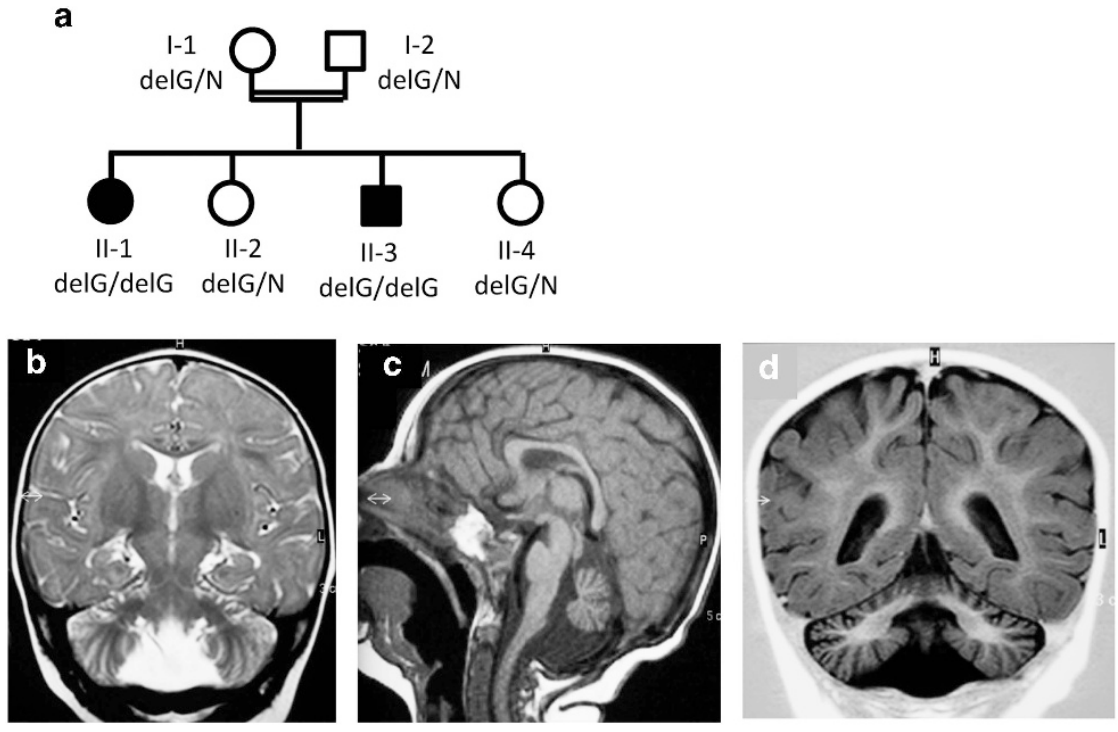

Figure 1 Family pedigree (filled symbols for affected members) with genotyping of the p.(Gly15Glufs*75) variant (del) in the QIL1/MIC13 gene (a). Brain MRI of patient II-1 (b, c) and patient II-3 (d) at 1 year of age: coronal T2-weighted image (b), sagittal T1-weighted turbo spin-echo image (c) and coronal T1-weighted image (d) disclosing small atrophic cerebellar hemispheres and vermis.

a

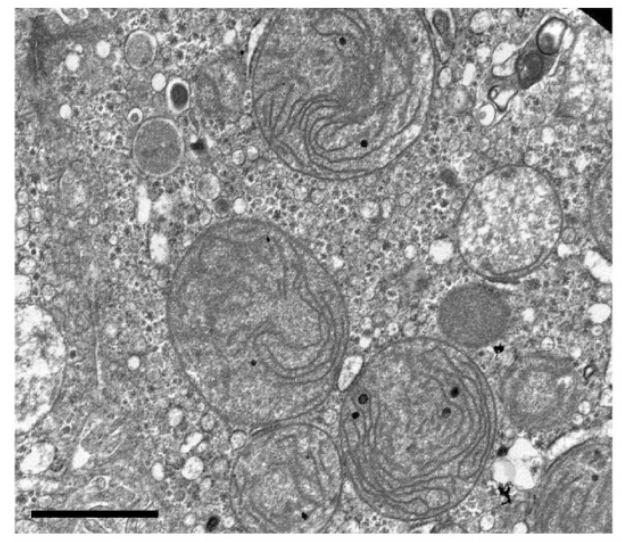

b

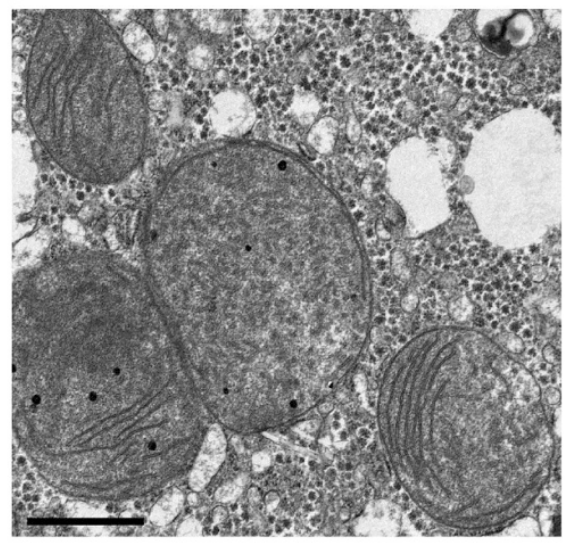

C

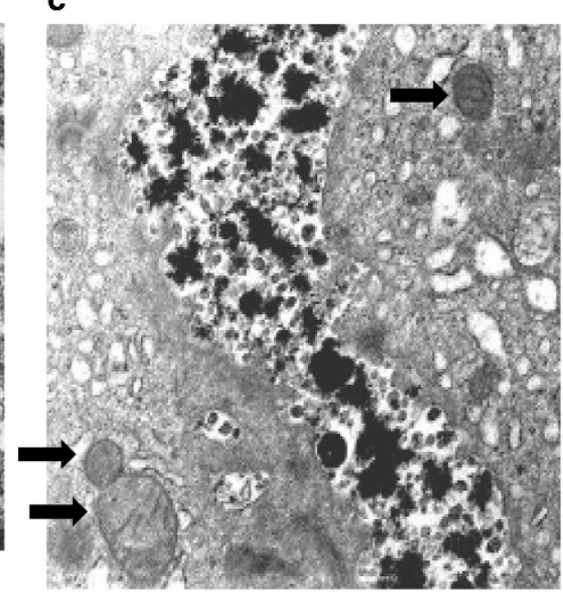

Figure 2 Electron microscopy of liver tissue of patient II-1 at 3 years of age: (a, b) representing images of enlarged mitochondria with abnormal distribution, packed cristae with curvilinear pattern and abnormal microtubular structures. Bar $-1 \mu \mathrm{m}$. (c) Normal mitochondria (arrows) in the liver of an infant with cholestasis (PFIC). ${ }^{19}$ 
visual function and no voluntary movements; the head circumference was unchanged $(<3$ rd percentile, $-4.6 \mathrm{SD})$. Brain MRI revealed cerebellar and vermis atrophy (Figure 1d). Echocardiography at 10 months revealed nonobstructive hypertrophic cardiomyopathy. The patient was admitted at 12 months with stupor and failure to feed and died 3 days later.

Throughout life both patients had impaired liver functions with peak levels of plasma alanine aminotransferase $434 \mathrm{U} / \mathrm{l}(\mathrm{N}<34 \mathrm{U} / \mathrm{l})$, aspartate aminotransferase $471 \mathrm{U} / 1 \quad(\mathrm{~N}<35 \mathrm{U} / 1)$, gamma glutamyl transpeptidase $454 \mathrm{U} / 1$ $(\mathrm{N}<33 \mathrm{U} / \mathrm{l})$ and abnormal coagulation (international normalized ratio 2.0 $(\mathrm{N}<1.3)$; bilirubin and alkaline phosphatase were normal as was plasma creatine kinase. Most mitochondrial fatty acid oxidation defects, peroxisomal biogenesis defects and protein glycosylation defects were excluded by the normal profile of acylcarnitines, very long-chain fatty acids and isoelectrofocusing of transferrins in plasma, respectively. Mitochondrial respiratory chain defect was suspected in both patients as plasma lactate levels were invariably increased to $4.0-6.0 \mu \mathrm{M}(\mathrm{N}<1.3 \mu \mathrm{M})$, alanine level was $464 \mu \mathrm{M}(\mathrm{N}<350 \mu \mathrm{M})$ and urinary organic acids analysis revealed increased excretion of 3-methylglutaconic and 3-methyglutaric acids and Krebs cycle metabolites. Muscle and liver biopsy were performed in patient II- 1 at 15 months and 3 years of age, respectively. Muscle histology was normal but in liver chronic inflammatory infiltration with bile canaliculi proliferation, fibrotic septa and focal macrovesicular fatty changes were noted. Electron microscopy of the liver tissue revealed abnormally shaped, spherical mitochondria with loss of the normal architecture of the cristae which was replaced by concentric stacks of inner membrane (Figures $2 \mathrm{a}$ and $\mathrm{b}$ ). The enzymatic activities of the mitochondrial respiratory chain complexes $1,2+3$ and 4 in liver homogenate were beneath the lower limit of control range when normalized to citrate synthase (CS) and decreased to $0.206 \mathrm{U} / \mathrm{U}$ CS (range 0.211-0.557), $0.068 \mathrm{U} / \mathrm{U}$ CS (range 0.117-0.231) and $0.73 \mathrm{U} / \mathrm{U}$ CS (range 0.83-1.82), respectively, whereas complex 2 activity was within the control range (Supplementary Table S1). In muscle mitochondria, these activities were within the normal range. The abnormal enzymatic pattern in liver tissue was suggestive of a defect in the synthesis of mitochondrial DNA (mtDNA) encoded proteins; however, in patient II-3 fibroblasts, the mtDNA/nuclear DNA ratio was within the normal range (128\% of the control mean) as were the activities of the mitochondrial respiratory chain complexes and mitochondrial morphology. Furthermore, in his blood, the mtDNA was of normal length suggesting that the mitochondrial respiratory chain defect observed in the liver tissue was not the result of a primary impairment in mtDNA replication or maintenance.

\section{Methods}

Homozygosity mapping. Homozygosity mapping was performed with DNA samples of the patients II-1 and II-3 and their healthy sister II-2. We used Genechip Human mapping $250 \mathrm{~K}$ SNP array (Affymetrix, Santa Clara, CA, USA) according to the manufacturer's protocol. Briefly, digestion with NspI, ligation of the adaptor, and amplification with a generic primer that recognizes the adaptor sequence were followed by fragmentation, end labeling and hybridization to the chip in accordance with the manufacturer's instructions. Homozygous regions $>2.5 \mathrm{Mb}$ were manually detected.

Whole-exome analysis. Exonic sequences were enriched in the DNA sample of patient II-3 using SureSelect Human All Exon 50 Mb Kit (Agilent Technologies, Santa Clara, CA, USA). Sequences were determined by HiSeq2000 (Illumina, San Diego, CA, USA) and 100-bp were read paired-end. Reads alignment and variant calling were performed with DNAnexus software (San Francisco, CA, USA) using the default parameters with the human genome assembly hg19 (GRCh37) as a reference. We then removed variants that resided on Chr.X, were called less than X8, were off-target, heterozygous, synonymous, MAF $>1 \%$ in Exome Aggregation Consortium (ExAC, Cambridge, MA, USA) or MAF $>4 \%$ at the Hadassah in house database. Parental consent was given for DNA studies and the study was performed with the approval of the ethical committees of Hadassah Medical Center and the Ministry of Health.

Study of MICOS complex in patient fibroblasts. Cultured skin fibroblasts of patient II-3 and a healthy control grown in RPMI supplemented with 20\% FBS and penicillin/streptomycin were lysed in RIPA buffer (50 mM Tris-HCl pH 7.5, $150 \mathrm{~mm} \mathrm{NaCl}, 1 \%$ sodium deoxycholate, $1 \% \mathrm{NP} 40,1 \%$ SDS, 1 mu EDTA) with
$1 \times$ Protease Inhibitor Mixture I (EMD Millipore, Merck KGaA, Darmstadt, Germany) and incubated on ice for $30 \mathrm{~min}$. Lysates were cleared by centrifugation at $13000 \mathrm{~g}$ for $10 \mathrm{~min}$ at $4{ }^{\circ} \mathrm{C}$. $6 \times$ Laemmli sample buffer was added and equivalent amounts of protein, determined by Bradford assay, were resolved by SDS-PAGE, transferred to nitrocellulose membrane (BioRad, Hercules, CA, USA) and immunoblotted with the following primary antibodies: $\alpha$-QIL1/MIC13 (Sigma, Darmstadt, Germany, SAB1102836); $\alpha$-MIC10 (Aviva, San Diego, CA, USA, ARP44801-P050); $\alpha$-MIC60 (Proteintech, Rosemont, IL, USA, 10179-1-AP); and $\alpha$-GAPDH (Sigma, G9545). Anti-rabbit IgG secondary antibody conjugated to DyLight 800 (Thermo Fisher Scientific, Waltham, MA, USA, PISA535571) was used and visualized with the Odyssey Infrared Imaging System (LI-COR, Lincoln, NE, USA). Linear adjustments to images were made using Adobe Photoshop.

\section{RESULTS}

\section{A frameshift variant in the QIL1/MIC13 gene segregates with disease}

The homozygosity mapping using samples of the two patients and their healthy sister yielded five regions where the patients, but not their unaffected sister, were homozygous for multiple long stretches of SNPs with identical genotype (Supplementary Table S2A). These regions, totally spanning $72.4 \mathrm{Mb}$ of genomic DNA, included 50 genes predicted to function within the mitochondria (MitoCarta2.0) (Supplementary Table S2A). We therefore performed exome analyses in patient II-3 DNA. This analysis yielded 53.75 million confidently mapped reads (mean coverage X63). Following reads alignment, variant calling and filtration as described in Methods, 10 homozygous variants remained within the linked regions (Supplementary Table S2B) but only 1 of them was also present in the MitoCarta2.0 list and segregated in the rest of the family as determined by Sanger sequencing. This was hg19 chr19:g.5679760 delC, NM_205767 c.44delC, p.(Gly15Glufs $\left.{ }^{\star} 75\right)$ in the C19ORF70 (QIL1/MIC13) gene. The two patients were homozygous for the variant and the parents and their two healthy daughters were heterozygotes (Figure 1a). The variant was not present in the ExAC database (accessed August $2015)$ or in the Hadassah database ( 650 ethnic matched patients).

\section{QIL1/MIC13 and MIC10 proteins are undetectable in patient fibroblasts}

QIL1/MIC13 encodes a 118 amino acid protein recently shown to be a constituent of the mature MICOS complex and required for MICOS complex stability and the stability of MICOS subunits, MIC10, MIC26 and MIC27, but not for the stability of MIC60, MIC19 and MIC25. ${ }^{8}$ The p. (Gly15Glufs $\left.{ }^{\star} 75\right)$ variant is predicted to encode a shorter, mostly missensed protein that is likely to be unstable and/or non-functional.

We examined the status of QIL1/MIC13 in cultured skin fibroblasts of patient II-3 by western blot analysis (Figure 3a). The analysis revealed that both QIL1/MIC13 and MIC10 were undetectable as compared with control fibroblasts extracts. In contrast, MIC60 was present in extracts from cultured skin fibroblasts of patient II-3 in a comparable abundance to that of the control. Thus, the MICOS complex phenotype in cells of patient II-3 is comparable to that observed in cells depleted of QIL1/MIC13 (Figure 3b and ref. 8).

\section{DISCUSSION}

The two described patients suffered from a lethal neurodegenerative disorder of infantile onset accompanied by hepatocellular dysfunction. Mitochondrial respiratory chain defects were a suspected cause based on increased plasma lactate and alanine levels, and abnormal urinary organic acid profile with 3-methylglutaconic aciduria and excessive excretion of Krebs cycle metabolites. This was confirmed by the finding of decreased activities of mitochondrial respiratory chain complex 1, 3 and 4 in the liver of patient II-1 (Supplementary 
Table S1). Exome analysis revealed that both patients had a homozygous frameshift variant in QIL1/MIC13, which resulted in the complete absence of the QIL1/MIC13 protein in the patient fibroblasts (Figure 3a).

QIL1/MIC13 is a recently described core component of MICOS complex (Figure 3b) and a structural ortholog of yeast Mic12. ${ }^{10}$ MICOS is proposed to act as a master regulator/integrator of IMM shape and organization. In human cells the MICOS complex forms from a stable MIC60-MIC19-MIC25 subcomplex that requires QIL1/ MIC13 for the binding of MIC10, MIC26 and MIC27. ${ }^{8}$ Depletion of QIL1/MIC13 in human cells by siRNA results in MICOS disassembly, accumulation of MIC60-MIC19-MIC25 subcomplex and loss of MIC10, MIC26 and MIC27 ${ }^{8}$ with associated transformation of normal cristae structure to abnormal stacking or onion-like inner membrane morphology. Consistent with a loss of QIL1/MIC13 function in our patient cells, both QIL1/MIC13 and MIC10 were undetectable, whereas MIC60 was present at normal abundance. In addition, stacked IMM disconnected from the IBMs were observed in the liver mitochondria of our patient - a characteristic phenotype similar to that observed in yeast and human cells lacking MICOS function. ${ }^{4-6,8,11-13}$

The cause of deterioration in our patients could be the mitochondrial respiratory chain dysfunction, which mainly affected the central nervous system, the liver and the heart. The respiratory growth defect observed in yeast strains lacking MICOS has been attributed to impaired organization and/or position of the respiratory complex III and IV. ${ }^{7}$ Cristae architecture may be important for producing an optimal distance between residing electron transporting chain complexes, specifically between complex III/IV supercomplexes, localized at lamellar regions, and ATP synthase oligomers localized to the curved edges. ${ }^{14-17}$

This is the first description of a defect in a MICOS subunit in human. As predicted from previously reported yeast observations our analysis shows that the MICOS assembly plays an essential role in mitochondrial respiratory chain function. QIL1/MIC13 deficiency joins the growing list of defects in IMM-organizing determinants. Of interest, these defects, including TAZ or DNAJC19 variants (resulting in cardiolipin remodeling defects) and SERAC1 variants

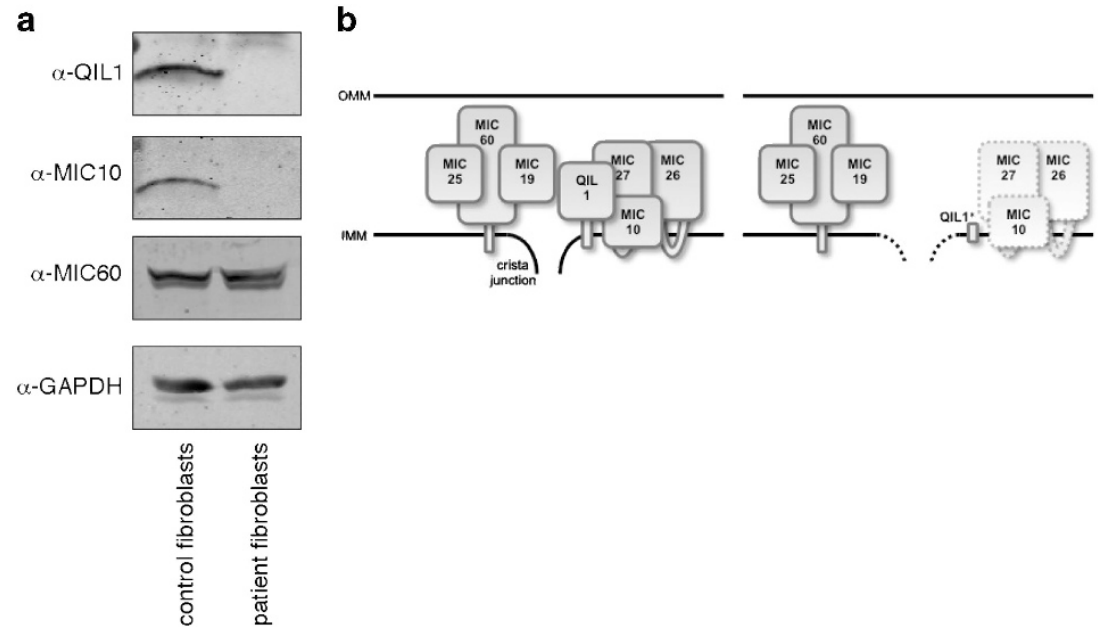

Figure 3 (a) QIL1/MIC13 frameshift variant causes loss of QIL1/MIC13 expression and destabilization of the MICOS complex. Representative western blot analysis with the indicated antibodies of whole-cell lysates from control (left) and patient II-3 (right) skin fibroblasts. (b) A schematic representation of the consequence of QIL1/MIC13 deficiency is shown. Left: The holo-MICOS complex, consisting of two subcomplexes (MIC60/MIC19/MIC25 in blue; QIL1/ MIC10/MIC26/MIC27 in red), is localized to cristae junctions in normal cells. Right: In the patients, QIL1/MIC13 is truncated and missensed and is unstable and non-functional, represented by QIL1/MIC13*. Loss of QIL1/MIC13 function leads to destabilization of the MIC10/MIC26/MIC27 subcomplex, indicated by dashed lines, while MIC60/MIC19/MIC25 remains intact. Loss of QIL1/MIC13 function causes loss or reduction in cristae junctions and altered cristae morphology. ${ }^{8}$ A full color version of this figure is available at the European Journal of Human Genetics journal online.

Table 1 Clinical presentation and genetic causes of 3-methylglutaconic aciduria

\begin{tabular}{lll}
\hline Gene & Clinical presentation & Additional laboratory findings \\
\hline TAZ & X-linked cardiomyopathy, short stature & Neutropenia \\
MNAJC19 & Mild intellectual disability, dilated cardiomyopathy, growth failure, & Anemia, elevated liver transaminases \\
CPA3 & Optic atrophy, cataract, spasticity & Hypoglycemia, lactic acidosis, elevated serum transaminase levels, \\
SERAC1 & Psychomotor retardation, spasticity or dystonia, & coagulopathy, hyperammonemia \\
TMEM70 & Sensorineural deafness & Lactic acidosis \\
AUH & hychomotor retardation, microcephaly, hypotonia, growth retardation, & \\
QIL1/MIC13 & Late onset leukoencephalopathy & Levere psychomotor retardation starting at $~$ \\
& intractable seizures, cerebellar atrophy, early death & Lacidemia, neutropenia, elevated liver transaminases
\end{tabular}


(resulting in mitochondrial phospholipid metabolism defect), ${ }^{18}$ are all associated with 3-methylglutaconic aciduria (Table 1).

\section{CONFLICT OF INTEREST}

The authors declare no conflict of interest.

1 Vogel F, Bornhövd C, Neupert W, Reichert AS: Dynamic subcompartmentalization of the mitochondrial inner membrane. J Cell Biol 2006; 175: 237-247.

2 Wurm CA, Jakobs S: Differential protein distributions define two sub-compartments of the mitochondrial inner membrane in yeast. FEBS Lett 2006; 580: 5628-5634.

3 Mannella CA, Marko M, Penczek P, Barnard D, Frank J: The internal compartmentation of rat-liver mitochondria: tomographic study using the high-voltage transmission electron microscope. Microsc Res Tech 1994; 27: 278-283.

4 Hoppins S, Collins SR, Cassidy-Stone A et al: A mitochondrial-focused genetic interaction map reveals a scaffold-like complex required for inner membrane organization in mitochondria. J Cell Biol 2011; 195: 323-340.

5 von der Malsburg K, Müller JM, Bohnert M et al: Dual role of mitofilin in 'mitochondrial membrane organization and protein biogenesis. Dev Cell 2011; 21: 694-707.

6 Harner M, Körner C, Walther D et al: The mitochondrial contact site complex, a determinant of mitochondrial architecture. EMBO J 2011; 30: 4356-4370.

7 Friedman JR, Mourier A, Yamada J, McCaffery JM, Nunnari J: MICOS coordinates with respiratory complexes and lipids to establish mitochondrial inner membrane architecture. Elife 2015; 4: e07739.

8 Guarani V, McNeill EM, Paulo JA et al: QIL1 is a novel mitochondrial protein required for MICOS complex stability and cristae morphology. Elife 2015; 4: e06265.
9 Bohnert M, Zerbes RM, Davies KM et al: Central role of Mic10 in the mitochondrial contact site and cristae organizing system. Cell Metab 2015; 21: 747-755.

10 Huynen MA, Mühlmeister M, Gotthardt K, Guerrero-Castillo S, Brandt U: Evolution and structural organization of the mitochondrial contact site (MICOS) complex and the mitochondrial intermembrane space bridging (MIB) complex. Biochim Biophys Acta 2015; 1863: 91-101.

11 Rabl R, Soubannier V, Scholz R et al: Formation of cristae and crista junctions in mitochondria depends on antagonism between Fcj1 and Su e/g. J Cell Biol 2009; 185: 1047-1063.

12 Weber TA, Koob S, Heide $\mathrm{H}$ et al: APOOL is a cardiolipin-binding constituent of the Mitofilin/MINOS protein complex determining cristae morphology in mammalian mitochondria. PLoS One 2013; 8: e63683.

13 John GB, Shang Y, Li L et al: The mitochondrial inner membrane protein mitofilin controls cristae morphology. Mol Biol Cell 2005; 16: 1543-1554.

14 Rieger B, Junge W, Busch KB: Lateral pH gradient between OXPHOS complex IV and F (0)F(1) ATP-synthase in folded mitochondrial membranes. Nat Commun 2014; 5: 3103.

15 Strauss M, Hofhaus G, Schroder RR, Kuhlbrandt W: Dimer ribbons of ATP synthase shape the inner mitochondrial membrane. EMBO J 2008; 27: 1154-1160.

16 Acehan D, Malhotra A, Xu Y, Ren M, Stokes DL, Schlame M: Cardiolipin affects the supramolecular organization of ATP synthase in mitochondria. Biophys J 2011; 100: 2184-2192.

17 Davies KM, Strauss M, Daum B et al: Macromolecular organization of ATP synthase and complex I in whole mitochondria. Proc Natl Acad Sci USA 2011; 108: $14121-14126$.

18 Wortmann SB, Kluijtmans LA, Rodenburg RJ et al: 3-Methylglutaconic aciduria - lessons from 50 genes and 977 patients. J Inherit Metab Dis 2013; 36: 913-921.

19 Iancu TC, Manov I: Electron microscopy of liver biopsies; in Takahashi H (ed.): Liver Biopsy. InTech, pp 109-136, ISBN 978-953-307-644-7. Available from http://www. intechopen.com/books/liver-biopsy/electron-microscopy-of-liver-biopsies.

Supplementary Information accompanies this paper on European Journal of Human Genetics website (http://www.nature.com/ejhg) 